\title{
Ecouter, voir : architectures du spectacle antique
}

\author{
Michel E. Fuchs
}

\section{Q OpenEdition}

Journals

Édition électronique

URL : http://journals.openedition.org/edl/127

DOI : $10.4000 /$ edl. 127

ISSN : 2296-5084

\section{Éditeur}

Université de Lausanne

\section{Édition imprimée}

Date de publication : 15 mai 2011

Pagination : 341-354

ISBN : 978-2-940331-25-3

ISSN : 0014-2026

\section{Référence électronique}

Michel E. Fuchs, «Ecouter, voir : architectures du spectacle antique », Études de lettres [En ligne], 1-2 I 2011, mis en ligne le 15 mai 2014, consulté le 20 décembre 2020. URL : http:// journals.openedition.org/edl/127 ; DOI : https://doi.org/10.4000/edl.127 


\section{ÉCOUTER, VOIR: ARCHITECTURES DU SPECTACLE ANTIQUE}

Au début était le culte de Dionysos, pour qui va s'élaborer le théâtre, le jeu lui-même comme le lieu où il se déroule. Si les Grecs portent le drame, l'action dans un lieu fixe, les Romains vont marquer de leur empreinte l'évolution et la diffusion du bâtiment du théâtre et plus généralement des édifices de spectacle. Ceux-ci se sont perfectionnés, les aménagements techniques se sont affinés, adaptés dès la fin de la République et sous l'Empire, au point que l'on compte aujourd'hui près d'un millier de théâtres connus, tous au bénéfice de traitements particuliers. Pour mieux en rendre compte, la recherche de modèles reste une préoccupation première, l'héritage grec, italiote ou étrusque méritant d'être débusqué dans le théâtre, l'amphithéâtre ou le cirque romain. Certaines constantes apparaissent cependant de l'Antiquité à nos jours. Il y a tout d'abord celui qui voit, le spectateur, qui constitue le "théâtre" selon Hérodote, l'assemblée qui regarde, selon l'étymologie grecque du terme; celui-là occupe la zone étagée des gradins. En face, il y a celui qui est vu sur un espace délimité, hors des coulisses qui servent aussi bien au vestiaire, à l'attente, à la préparation qu'à faire sortir un personnage de lieux cachés aux spectateurs comme de l'intérieur des maisons dans les comédies de Ménandre. Une cloison sépare les coulisses de la scène, cloison nécessairement ouverte pour permettre les apparitions et les disparitions des acteurs. Un tel aménagement implique une connivence entre le public et les acteurs, des conventions à la base de l'illusion théâtrale ${ }^{1}$ : le plateau de la scène et l'arène, nus ou aménagés, sont les

I. «L'illusion... suppose entre le spectateur et l'image scénique un rapport tel que le spectateur croie à la réalité de l'action scénique et de son cadre, que le monde 
lieux où se déroule l'action; au théâtre, l'acteur, costumé ou non, est un personnage fictif. Dans l'arène, le réel est mis en scène. Au théâtre, le public, spectateur, auditeur, admet l'invraisemblable, jusqu'à l'absence même de décors, d'images, qu'elles soient portées par l'acteur ou par la scène. Il descend aux Enfers avec Dionysos déguisé en Héraclès dans les Grenouilles d'Aristophane, le chœur des grenouilles signifiant le passage de l'Achéron, lançant son Brekekex koax koax sur le marais infernal. Il est devant le mur de Troie, écoutant les lamentations de Cassandre sur une scène totalement vide, seulement occupée par un coin de cendres fumantes et par un drap informe, voyant évoluer, gesticulante, une actrice sans masque, livide, en simple robe, dans la très contemporaine Cassandre de Michael Jarrel. Au bord de l'arène, il assiste aux chasses, aux supplices, aux combats et aux courses. Si nous pouvons aujourd'hui non seulement lire mais aussi comprendre, imaginer, un spectacle antique ou une pièce d'Harold Pinter, ce n'est pas seulement une question de familiarité avec le langage utilisé; interviennent les principes d'organisation du spectacle, les fondements de la mise en scène, le parti pris architectural.

Est-il donc possible de dégager de l'étude des édifices antiques du spectacle les principes qui ont conduit à une architecture donnée? Le théâtre grec et le théâtre romain ont pourtant évolué du VI $\mathrm{I}^{\mathrm{e}}$ siècle av. J.-C. au IV siècle apr. J.-C., entre naissance et abandon. Des changements dans le jeu autant que dans les structures sont apparus. Ce ne sont pas les quarante-cinq pièces complètes du théâtre grec qui suffisent à nous faire prendre conscience de la production suscitée par dix siècles de spectacles. C'est pourtant bien cette question essentielle qui sous-tend aujourd'hui l'étude des bâtiments qui les ont vus naître: quels types de spectacles y pratiquait-on? Ceux des provinces équivalaient-ils à ceux de la Capitale? Quel sens recouvraient-ils pour leurs initiateurs, éditeurs et évergètes? Que dire du cadre religieux et politique dans lequel ils s'inséraient?

Loin de nous l'idée que les interventions réunies ici répondent aux nombreuses questions que suscitent les bâtiments du spectacle dans l'Antiquité. Elles sont d'abord issues de deux journées réservées aux

imaginaire devienne pour lui un monde réel, même si en entrant au théâtre il a pleinement conscience des conventions théâtrales ", D. Bablet, Esthétique générale du décor de théâtre de 1870 à 1914, p. 15. 
doctorants des universités de Lausanne, Genève, Neuchâtel et Fribourg, dans le cadre de l'Ecole doctorale sur les sciences de l'Antiquité des universités de Suisse romande (EDOCSA). La réunion de spécialistes a suscité des communications variées, dont une majorité traite certes d'édifices de nos régions, mais qui ont pourtant comme point commun l'interrogation du contenant pour mieux aborder son contenu. Le but était aussi d'exposer quelques points nouveaux et l'aboutissement de certaines réflexions, tout en offrant aux doctorants l'occasion de présenter leurs travaux à des connaisseurs.

Pour parler théâtre, il faut avant tout en déterminer la forme. Les fouilles archéologiques livrent leur comptant d'informations qu'il s'agit d'interpréter non seulement intrinsèquement, mais aussi en regard du contexte particulier à chaque site. Bâtiment souvent connu de longue date, le théâtre ou l'amphithéâtre a une histoire contemporaine aussi chargée que celle qui l'a vu naître et se modifier. Le théâtre d'Alésia allie un contexte symboliquement fort à une série de restaurations $\mathrm{du} \mathrm{XX}^{\mathrm{e}}$ siècle qui en rendent la lecture délicate. La fouille et l'analyse minutieuse des vestiges nécessitent une description attentive avant d'en dégager et d'en comprendre le plan. A l'exploitation d'une carrière succèdent des assainissements du terrain qui le préparent vraisemblablement à recevoir très tôt des spectateurs; un projet de théâtre voit le jour suivi d'un premier édifice entre le début et la fin du Ir siècle apr. J.-C. L'ensemble est détruit puis reconstruit dans le courant du $\mathrm{II}^{\mathrm{e}}$ siècle apr. J.-C. Découvert en 1998, le théâtre de Lousonna-Vidy a droit lui aussi à une approche attentive pour comprendre un peu de son organisation. Il est bizarrement orienté au sud, ce qui s'explique par la nature du terrain dans lequel il est implanté; il faudrait cependant pouvoir étendre la fouille du secteur pour dire si nous avons affaire à un théâtre isolé en bordure du bourg antique ou si nous sommes plutôt devant un édifice lié à un sanctuaire en limite d'agglomération. Sa petite taille $(60 \mathrm{~m})$ par rapport aux trois autres théâtres connus en Suisse, à Augst, Avenches et Lenzburg, s'accorde aux dimensions du vicus. Usant de la pierre locale, la molasse, pour ses gradins munis de banquettes et de marchepieds, sa cavea, le lieu où se réunissent les spectateurs, est plus plate que celle des autres théâtres et offre une structure polygonale. L'orchestra au pied des gradins, ce demi-cercle réservé à l'origine à l'évolution des chœurs ici prolongé de $11 \mathrm{~m}$, est occupée par les restes d'une scène quadrangulaire dont le dispositif ne devait guère se différencier des théâtres modernes 
à sol horizontal. Datée du début du II siècle apr. J.-C., la construction se rapproche des théâtres ruraux plutôt que de ceux de grandes villes comme Avenches ou Augst. Dégagé lui aussi récemment, entre 1985 et 2008, le théâtre de Dalheim, dans le grand-duché du Luxembourg, apporte des compléments précieux à l'étude du théâtre dit gallo-romain: comme à Alésia, il est érigé sur une zone de carrière, sa première phase de construction datant du début du $\mathrm{II}^{\mathrm{e}}$ siècle apr. J.-C.; il est muni de gradins en bois et d'un mur de cavea en pierre. Celui-ci a été renforcé en sous-œuvre dans les angles à titre préventif et non à la suite de dégâts comme on l'avance fréquemment pour ce genre d'interventions. Dans une phase tardive, des banquettes de pierre ont été aménagées avec accoudoirs aux extrémités des deux premiers rangs bordant l'orchestra. Une proédrie, ces sièges destinés aux autorités et aux privilégiés, est ainsi pour la première fois attestée au nord des Alpes. De plus, deux dalles de fondation dans l'orchestra invitent à y restituer deux autels, phénomène connu par ailleurs ${ }^{2}$. Une voie longera le mur de la cavea, dont la fouille a montré que le secteur sud a été implanté dans des remblais d'ordures et de matières fécales. Le théâtre est détruit dans les années 270 apr. J.-C., puis réoccupé du $\mathrm{IV}^{\mathrm{e}}$ au début $\mathrm{du} \mathrm{V}^{\mathrm{e}}$ siècle, selon une évolution observée à Avenches. Un tel développement n'est pas si différent de celui du théâtre de Lillebonne en Haute-Normandie, dont la fonction scénique est abandonnée à la fin du III e siècle avant une réutilisation des lieux attestée par de nombreux foyers jusque dans la première moitié du $\mathrm{V}^{\mathrm{e}}$ siècle. L'édifice doit son identification précoce au comte de Caylus, le célèbre "antiquaire acariâtre et brusque" des vers de Diderot en 1767. La conséquence d'une telle reconnaissance est une suite d'interventions dès le début du XIX siècle jusqu'aux fouilles récentes de 2007-2009. Avant de livrer les résultats des dernières campagnes, l'historique des recherches s'avère ici nécessaire, accompagné d'une étude pointue des archives à disposition. L'approche est incontournable lorsqu'il s'agit d'interpréter les plans anciens, les changements apportés au monument, les choix de présentations et les interprétations qui en modifient la structure. Avec ses 106,50 m de dimensions générales, sans les contreforts, le théâtre de Lillebonne fait partie des grands monuments du genre et, par sa grandeur même, suscite des interrogations sur le devenir de tels ensembles architecturaux. Trois théâtres en offrent l'éventail des cas possibles, du

2. Voir J.-C. Moretti (éd.), Fronts de scène et lieux de culte dans le théatre antique. 
théâtre de Toulouse connu par les seuls documents du XIX ${ }^{\mathrm{e}}$ siècle au monument d'Orange dont la conservation du bâtiment de scène sur $36 \mathrm{~m}$ de hauteur a permis d'en restituer la couverture, en passant par les maigres vestiges de murs de cavea du théâtre de Marseille.

L'ampleur du monument, voilà qui pose souvent sinon toujours problème. Le théâtre de Mandeure le démontre et suscite la mise en place d'une méthode d'analyse rigoureuse, d'une prudence dans l'interprétation des résultats, d'une distance par rapport aux acquis anciens. Lorsque le mobilier archéologique fait défaut, c'est l'architecture qu'on interroge. Lauscultation des phases de construction aboutit ainsi à clarifier non pas la datation de l'édifice, mais l'évolution de ses aménagements: rehaussement des sols, blocs en réemploi, dont deux gradins inscrits, un nom, un numéro de place. L'orchestra se fait semi-circulaire et outrepassée, les murs s'élèvent, par étapes. Des arcades et des colonnes rythment les façades, un fond de scène barre le dégagement sur le sanctuaire auquel fait face le théâtre. Ce n'est donc pas la vision sur le lieu de culte du dieu qui importe, mais le jeu qu'il suscite. Si tout indique à Mandeure que l'on doit écarter la définition du bâtiment comme étant de type galloromain, ce serait précisément à ce type que se référerait le théâtre de Genainville. Il adopte en tout cas une décoration architecturale dont la richesse n'a rien à envier à celle du temple qui lui répond, dans un foisonnement typique de cette peur du vide si caractéristique de l'art romain, horror vacui exacerbé sous les empereurs Sévères. Pourtant, l'étude des structures indique qu'il était impossible de juger de l'ornementation du temple depuis le théâtre. Tout au plus les spectateurs des derniers gradins, l'équivalent du poulailler d'aujourd'hui, avaient quelque chance de voir le temple; ceux qui étaient assis dans la proédrie ne l'avaient pas. C'est que l'enjeu se situait ailleurs. Dans le cas d'Avenches, la relation entre temple et théâtre est indéniable, du moins du point de vue de l'axialité, de la largeur identique des deux monuments et des proportions de l'ensemble (fig. 1). L'espace est même défini de telle manière qu'il marque une délimitation nette entre l'extérieur et l'intérieur de ce que l'on pourrait assimiler à un enclos sacré, d'autant qu'il se voit bordé de nouveaux temples à la fin du $\mathrm{II}^{\mathrm{e}}$ siècle apr. J.-C. Depuis le centre de la cavea, le temple du Cigognier devait être visible du portique et des gradins supérieurs du théâtre, par-dessus le mur de fond de scène; à travers la porte centrale ouverte dans le même mur, depuis les tout premiers rangs au niveau de l'orchestra, devaient poindre l'entrée principale 
Fig. 1 - Vue d'un spectateur debout dans l'axe du théâtre d'Avenches en direction du sanctuaire du Cigognier, depuis le portique en haut des gradins (à gauche) et depuis l'orchestra (à droite).

et l'escalier monumental du sanctuaire ${ }^{3}$. C'est de là que partaient sans doute les processions se rendant à l'amphithéâtre les jours de fête en l'honneur de l'empereur. Sur la colline médiévale d'Avenches, l'édifice dominait la ville antique et reflétait la hiérarchie de la société locale. Des aménagements spécifiques étaient là pour le souligner, dont un couloir de service en bordure d'arène fonctionnant aussi bien pour les effets du spectacle que pour rejoindre le rang d'honneur au sud du monument. A l'est, une terrasse surmonte la porte d'entrée dans l'arène; elle était sans doute réservée à l'installation des effigies de divinités et d'empereurs qui avaient participé au cortège d'ouverture des jeux. L'importance grandissante de cette procession, qui illustrait aussi bien l'ordre social dans la cité et ses environs que le lien avec l'Empire, se manifeste par la monumentalisation, peu après 165 apr. J.-C., de l'extérieur de la cavea et de l'accès principal à l'amphithéâtre, transformé en une sorte d'arc de triomphe.

Pour en arriver à cet éloge imposant rendu aux victoires et au salut de l'empereur, il a fallu passer par diverses tentatives. L'une d'elles serait magnifiquement inscrite dans la forme de l'amphithéâtre de Leptis Magna: au lieu d'adopter l'ellipse caractéristique de la plupart des monuments consacrés aux combats d'animaux et de gladiateurs, celui-ci opte pour deux extrémités semi-circulaires et des raccords rectilignes. Peut-on cependant parler dans ce cas de tâtonnements? La démonstration est ici faite que, grâce à la date de l'inauguration de l'édifice, connue par une inscription (56 apr. J.-C.), grâce à la reprise des textes anciens, le plan choisi par l'architecte du monument de Tripolitaine annonce et illustre

3. G. Matter, Das römische Theater von Avenches/Aventicum. Architektur, Baugeschichte, kulturhistorische Aspekte, p. 170-177, fig. 159-160. 
l'amphithéâtre en bois du Champ de Mars à Rome, projeté par Néron en 57 apr. J.-C. Prendre l'option d'accoler deux théâtres par leurs scènes répond à une fonction: adapter le lieu à un nouveau type de spectacle, total, polyvalent, voulu par l'empereur, réalisé dans les neronia; la danse et la musique, les compétitions athlétiques et hippiques y disputaient l'arène aux chasses et aux combats de gladiateurs. La forme retenue satisfaisait pleinement l'acoustique et la vision, pas suffisamment pour survivre à Néron. Effet de la romanisation ou adaptation romaine aux conditions locales, les édifices de spectacle des provinces de Maurétanie sont eux aussi marqués par une certaine originalité dans leur traitement. A Caesarea, le roi Juba II, qui a passé son enfance à Rome, offre à sa ville un théâtre et un amphithéâtre entre 25 et 15 av. J.-C. à l'image de ceux qu'il a fréquentés dans la Capitale; les plus anciens d'Afrique, ils s'inspirent du théâtre de Pompée et de celui de Marcellus d'un côté, des gradins montés sur le forum romain de l'autre, s'il ne faut pas plutôt penser à l'influence du stade grec. A Tipasa, dans la province césarienne, plus de deux cents ans plus tard, un théâtre classique prend place, mais avec une scène hypertrophiée, suivi par un amphithéâtre dont l'arène adopte l'ellipse traditionnelle, mais dont la cavea est enfermée dans un plan quasi rectangulaire. En Tingitane, les villes de Lixus et de Zilil sont pourvues chacune d'un amphithéâtre à l'arène circulaire et d'un haut mur de podium pour le premier monument, dans une structure bien plus favorable à la représentation de chasses qu'aux combats de gladiateurs. Est-ce là une forme voulue par les citoyens ou faut-il envisager la présence de militaires à l'origine d'un si curieux aménagement jouxtant des thermes dans le cas de Lixus? ${ }^{4}$ Plus haut dans le temps, l'empreinte du pouvoir est indéniable sur le théâtre de Babylone. Celui-ci a été instauré par Alexandre le Grand, au centre de son empire, complété par une palestre pour en faire le lieu de convergence de la population grecque de la cité. Le théâtre est modifié, augmenté d'un mur de scène dans le deuxième quart du $\mathrm{II}^{\mathrm{e}}$ siècle av. J.-C., puis restauré par un Dioscourides au $\mathrm{II}$ siècle apr. J.-C. Comme nous l'apprennent les sources épigraphiques, les citoyens helléniques se réunissaient au théâtre pour certaines de leurs assemblées, à l'occasion de la lecture des lettres du roi et des gouverneurs. Le monument est désigné en akkadien et par un idéogramme comme la «maison de l'observation", traduction littérale du grec theatron. Lieu de

4. G. Hallier, «Un amphithéâtre militaire à Lixus?». 
réunion privilégié pour l'écoute citoyenne des annonces et des décrets officiels, il est aussi le lieu de diffusion par excellence de la culture grecque sous l'empire séleucide, parthe puis romain.

La forme caractéristique du théâtre antique est pour nous celle du demi-cercle outrepassé. Elle n'apparaît pourtant pas avant la fin du IVe siècle av. J.-C. Dans la capitale de la culture hellénique qu'est Athènes, le drame se jouait à l'origine sur l'agora de l'acropole, dans une orchestra non pas circulaire mais trapézoïdale entourée de gradins rectilignes. Au Ve siècle av. J.-C., le théâtre de Dionysos adoptait luimême ce plan. Le théâtre est alors ressenti non pas comme un édifice mais comme un espace où se déroule une performance que suivent des spectateurs. Le théâtre, c'est l'orchestra, une place vide, libre pour l'expression de la collectivité, comme l'agora. En Gaule, le théâtre d'Alba, dans l'ancien chef-lieu des Helviens en Ardèche, permet lui de remonter aux premiers édifices de type dit "gallo-romain", que l'on ferait mieux d'appeler "gaulois». Erigé vers 20 av. J.-C., il présente une cavea polygonale à double pente, la partie inférieure étant plus vaste, avec des gradins classiques, destinée à un public romanisé, la partie supérieure plus courte pour un public gaulois assis en tailleur sur des gradins en bois. Le devant de la scène est tronqué par rapport aux exemples grecs et romains, caractéristique qui a dû servir à la latinisation du peuple celtique, dans un espace que l'on peut considérer comme transculturel. En 15 apr. J.-C., le phénomène est intégré et le théâtre devient latin, rénové vers 120 apr. J.-C. L'originalité du premier édifice conduit à se demander quel répertoire devait y être joué. Les textes font certes défaut, mais le détour par les lettres étrusques et leurs livres sacrés, par les écritures gauloises et les dialectes celtiques toujours vivaces au IV siècle apr. J.-C., laisse supposer la transmission d'un message alliant la langue latine à la culture locale. Le premier théâtre d'Alba se révèle ainsi le prototype des monuments des Trois Gaules où se disaient les textes transposés du vieux fond celtique. Il ne faudrait toutefois pas écarter de cette réflexion l'existence des spectacles dansés et musicaux qu'offraient certainement les édiles à la communauté. Sur le modèle d'Auguste et du théâtre de Marcellus, synthèse d'influences diverses, autant signe de réconciliation entre les projets de César et de Pompée que rattaché à Apollon et outil 
de propagande impériale destiné aux masses ${ }^{5}$, l'élite gauloise a su tirer parti des édifices de spectacle construits dans ses cités, sans en oublier l'essentielle fonction ludique. A cela, il faut ajouter l'aspect religieux. En Gaule, les théâtres sont pour une bonne partie d'entre eux intégrés à des sanctuaires, que ce soit en ville, en bordure de la cité ou sur son territoire; ils ont alors les caractéristiques de la série dite gallo-romaine, soit le plateau scénique occupant l'orchestra, l'arrière-scène adossée au mur de scène et des entrées d'honneur ouvertes sur les côtés du mur rectiligne. Une seconde série s'insère dans la trame urbaine des agglomérations plutôt que de s'en détacher. Si la Gaule Narbonnaise, romanisée de plus longue date, a opté pour des solutions proches des ensembles italiens, si les choix sont variables dans les Trois Gaules, les théâtres sont tous intégrés à un circuit religieux qui relie les points de rassemblement de la population. Les amphithéâtres quant à eux sont régulièrement logés hors du réseau urbain, au point qu'ils manifestent par leur monumentalité le statut de la ville. Ils possèdent une chapelle réservée aux gladiateurs, dédiée à Hercule, à Mars ou à Diane, le plus souvent à Némésis à partir du II ${ }^{\mathrm{e}}$ siècle apr. J.-C., elle la maîtresse des caprices de la destinée, devenue la déesse de l'arène. Dès l'époque flavienne, après l'inauguration du Colisée, l'amphithéâtre a tendance à se substituer au théâtre: il jouit d'une bonne desserte routière, il s'aligne sur le forum, il s'associe à un sanctuaire. Les spectacles eux-mêmes s'inscrivent dans des calendriers, le public y assiste en rangées ordonnées, le gradin d'honneur se garnit. La répartition des édifices dans les Trois Gaules montre l'amphithéâtre généralement réservé aux chefs-lieux, le théâtre dit gallo-romain aux sanctuaires et aux agglomérations. Si les jeux offerts dans l'amphithéâtre sont aujourd'hui mieux connus, ceux du théâtre n'ont pas livré tous leurs secrets. Le développement des théâtres "gallo-romains» se fait au moment où Auguste découvre l'énorme potentiel rassembleur des jeux, quand la pantomime, à son invite, s'impose comme un spectacle total et unificateur. La solution gauloise répondrait ainsi parfaitement au nouveau genre choyé par l'empereur, un acteur évoluant sur la courte scène, accompagné de chanteurs et de musiciens qui occuperaient l'orchestra.

Les grandes fêtes données à l'occasion des jeux animent donc tout un paysage urbain, les édifices de spectacle, les places et les temples

5. R. Sablayrolles, «Espace urbain et propagande politique: l'organisation du centre de Rome par Auguste (Res Gestae, 19 à 21) », en part. 75-76. 
fournissant le cadre civique dans lequel les principes de Rome sont traduits en termes régionaux. Afin de faciliter entrées, sorties et installation des participants, des améliorations techniques sont régulièrement apportées aux monuments. Dans l'arène, la symbolique est mise en scène, la porte de la vie est celle du triomphe d'un côté de l'amphithéâtre, la porte de la mort est l'issue d'en face. Les chasses d'animaux, les venationes, sont de véritables chorégraphies bénéficiant d'apparitions isolées puis multiples grâce au perfectionnement des monte-charges dans des édifices comme le Colisée, grâce aux couloirs et à l'ordonnance des pièces de service, des carceres bordant l'arène ailleurs. Reflet de la société, théâtres et amphithéâtres sont de formidables outils de la propagande impériale. Ils réunissent les masses et mettent en présence le peuple et ses autorités politiques et religieuses. Hauts lieux émotionnels, ils reconstituent l'unité d'une foule plurielle lors de la décision sur le sort d'un gladiateur ou la reprise d'une scène. Le gladiateur thrace, l'essédaire gaulois sur son char et l'hoplomaque grec illustrent les victoires de l'empereur, les condamnés à mort sa justice, pour qu'ainsi meurent les ennemis de la république. Les jeux célèbrent l'étendue de son pouvoir, le triomphe de la civilisation romaine. Ils sont placés sous l'autorité des dieux, garants de la paix.

Panem et circenses, le mot de Juvénal dans ses Satires (10.81) résume depuis longtemps la portée des jeux et des édifices qui les abritent dans le monde romain. Le cirque, destiné avant tout aux courses de chars, a de quoi s'imposer par sa monumentalité et par la quantité de personnes qu'il draine aux jours de fête. Il participe aux origines de Rome avec le circus étrusque de Tarquin l'Ancien, à la fin d'un empire avec l'hippodrome byzantin de Constantinople. Il reprend aujourd'hui un peu de son lustre grâce à des projets interdisciplinaires qui ont pour point de départ l'étude du plus colossal de la série, le Cirque Maxime. C'est l'occasion d'en préciser le vocabulaire - le mur-barrière axial au centre de la piste n'est pas la spina mais l'euripe -, de revoir certains aménagements et leur évolution, dont l'usage abondant de l'eau, celle que les sparsores jetaient à la bouche et aux naseaux des chevaux, de refaire enfin le tour des cirques connus.

Si maquettes et modèles 3D sont une contribution précieuse à la compréhension de monuments marquants du paysage antique, les sons et les mouvements qu'ils enrobaient ne peuvent aujourd'hui qu' être 


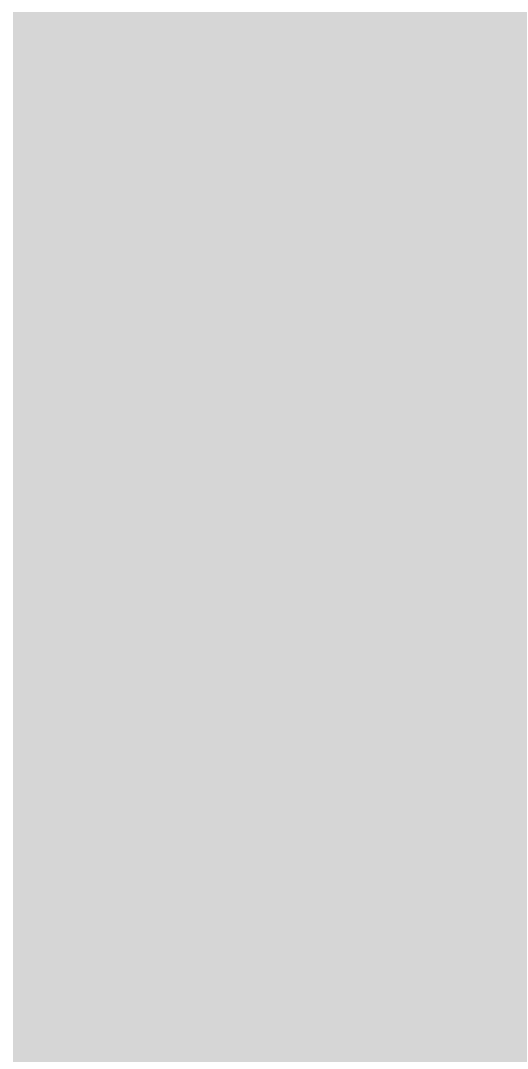

Fig. 2 - Dovecus, l'acteur tragique d'Avenches du début du III $^{\text {e }}$ siècle apr. J.-C. effleurés par les textes et les décors qui s'y rapportent (fig. 2) ${ }^{6}$. Au milieu du II ${ }^{\mathrm{e}}$ siècle av. J.-C., Polybe fait le récit des funérailles d'un aristocrate romain (6.53): il est porté vers le forum, présenté debout devant les rostres. Un fils adulte ou un proche énonce alors depuis les rostres les vertus et actions du défunt. Ainsi, celui-ci n'est plus pleuré par ses seuls proches mais touche l'ensemble du peuple. Un masque a été tiré du mort, reproduisant fidèlement ses traits; on le porte dans les sacrifices publics et, lorsqu'un membre de la famille meurt, un homme de la même stature et de même apparence générale que le défunt va s'affubler non seulement de son masque mais aussi de ses vêtements officiels. L'homme va ensuite parader sur un char en portant les insignes des charges de l'ancêtre. Puis, arrivés aux rostres, tous les hommes qui remplissent le rôle d'un ancêtre s'assoiront en rang sur des chaises en ivoire. "Quel glorieux spectacle (theama), dit Polybe, pour un jeune homme qui aspire à la réputation et à la noblesse!». Les funérailles deviennent théâtre politique: discours, dialogue, drame, acteurs, costumes, masques, cortège, scène. Le forum de Rome est ainsi le lieu des premières pièces "littéraires». Aux funérailles de Paul Emilien, à celles de César, un joueur de flûte et un joueur de trompette ou un corniste accompagnent un chœur de femmes chantant des lamentations sous la direction de la praefica, la pleureuse professionnelle. Vers

6. N. Savarese (a cura di/ed.), In scaena. Il teatro di Roma antica/The theater in ancient Rome; E. Rosso, "Le message religieux des statues divines et impériales dans les théâtres romains. Approche contextuelle et typologique». 
160 apr. J.-C., dans ses Métamorphoses (3.2-11), Apulée raconte le charriage de son héros dans la ville de Larissa en Thessalie. Le jour de la fête du dieu Rire, Lucius est accusé de meurtre devant un tribunal qui se réunit sur le forum de la ville. Face à l'affluence et à l'importance du procès, le public est invité à se rendre au théâtre. "Le peuple, nous dit Apulée, remplit avec une rapidité extraordinaire tout l'espace destiné aux spectateurs (caveae conseptum)»; voilà qui n'est pas sans faire penser aux aménagements perfectionnés d'un théâtre du $\mathrm{II}^{\mathrm{e}}$ siècle. "Même les entrées (aditus) et le toit étaient remplis à craquer, beaucoup grimpaient aux colonnes, d'autres s'accrochaient aux statues, un bon nombre se montraient à demi et à travers des lucarnes ou des caissons des combles, et tous, dans leur étonnant désir de voir (miro studio visendi), ne se souciaient pas du danger mortel qu'ils couraient ». A l'occupation forcenée des gradins s'ajoute celle des autres espaces du théâtre, dans une vision qui rappelle les architectures peintes peuplées de personnages de la Domus Aurea de Néron à Rome ou plus tard celles d'Ostie, à la période antonine. Le danger de chutes fait lui référence aux anecdotes connues à ce propos sous l'Empire. "Alors, les huissiers de la ville (publica ministeria) me font avancer à travers la scène (per proscaenium medium) comme une victime et m'installent au milieu de l'orchestre (orchestrae mediae)». L'aspect religieux lié à un théâtre est là clairement évoqué: au début d'une prestation, un sacrifice est accordé aux dieux après que la victime a été présentée à l'assemblée. Une fois tue la «voix terrible» (immanem vocem) de l'accusateur, Lucius fait son plaidoyer larmoyant qui déclenche l'hilarité des spectateurs. Après une ultime péripétie - l'arrivée à travers le théâtre (per medium theatrum) de deux pleureuses vêtues de noir, élevant «une clameur funèbre, accompagnée de hurlements»-, le héros est menacé de tortures et finalement découvre que les personnes qu'il aurait tuées sont en fait des outres qu'il a percées, de nuit et en état d'ivresse. Le rire éclate alors franchement, certains poussant même des cris de paon, d'autres se tenant le ventre. «Et c'est au milieu de la gaieté générale que tous quittent (en se pressant, facessunt) le théâtre en se retournant dans ma direction"; le verbe utilisé est tout à fait approprié à la fonction des vomitoires. Lucius quant à lui reste un moment pétrifié, «aussi glacé que n'importe lesquelles des statues ou des colonnes du théâtre»; c'est la dernière évocation du théâtre de Larissa par Apulée, une fois les gradins libérés des spectateurs. Peu de textes donnent autant d'indications sur le monde du théâtre romain, de plus à l'époque où ceux-ci connaissent 
leur plus grande expansion. L'exploration des textes antiques, des images et des édifices de spectacle eux-mêmes est loin d'être achevée. C'est en osant la confrontation avec de tels édifices que s'ouvrent de nouvelles perspectives, seules à même d'affiner nos connaissances et de combler l'espace vide laissé par les grands monuments du spectacle antique.

Michel E. Fuchs Institut d'Archéologie et des Sciences de l'Antiquité, Université de Lausanne 


\section{BIBLIOGRAPHIE}

BABlet, Denis, Esthétique générale du décor de théâtre de 1870 à 1914, Paris, CNRS, 1965.

Hallier, Gilbert, "Un amphithéâtre militaire à Lixus?", in VIII ${ }^{e}$ colloque international sur l' histoire et l'archéologie de l'Afrique du nord, éd. Mustapha Khanoussi, Tunis, Ministère de la Culture, 2003, p. 351-380.

Matter, Georg, Das römische Theater von Avenches/Aventicum. Architektur, Baugeschichte, kulturhistorische Aspekte, Lausanne, Cahiers d'archéologie romande, 2009 (Cahiers d'archéologie romande 114, Aventicum XV).

Moretti, Jean-Charles (éd.), Fronts de scènes et lieux de culte dans le théâtre antique, Lyon, Maison de l'Orient et de la Méditerranée, 2009 (Travaux de la Maison de l'Orient et de la Méditerranée 52).

Rosso, Emmanuelle, "Le message religieux des statues divines et impériales dans les théâtres romains. Approche contextuelle et typologique", in Fronts de scène et lieux de culte dans le théatre antique, éd. Jean-Charles Moretti, Lyon, Maison de l'Orient et de la Méditerranée, 2009, p. 89-126 (Travaux de la Maison de l'Orient et de la Méditerranée 52).

Sablayrolles, Robert, «Espace urbain et propagande politique: l'organisation du centre de Rome par Auguste (Res Gestae, 19 à 21)", Pallas 17, 1981, p. 59-77.

Savarese, Nicola (a cura di/ed.), In scaena. Il teatro di Roma antica/The theater in ancient Rome, Milano, Electa, 2007.

\section{Crédits iconographiques}

Fig. 1:

Restitution infographique ProSpect, Georg Matter.

Fig. 2:

Photo Musée romain d'Avenches. 


\section{ANNEXES}

Georg Matter, Le théâtre romain d'Avenches: quelques réflexions sur sa situation et sa fonction dans l'ensemble "temple du Cigognier/théâtrel temples au Lavoëx»

Le temple du Cigognier et le théâtre représentent deux éléments d'un même complexe architectural, que révèlent l'alignement axial et la largeur identique des deux monuments. En l'état actuel des connaissances, les éléments de datation archéologique disponibles parlent clairement en faveur d'une période de construction qui s'étend, pour les deux bâtiments, de la fin du $\mathrm{I}^{\mathrm{er}}$ siècle au $1^{\mathrm{er}}$ tiers du $\mathrm{II}^{\mathrm{e}}$ siècle apr. J.-C. Cette simultanéité, attestée par la datation du matériel archéologique, est corroborée par l'analyse de la disposition générale du complexe, qui montre des proportions parfaites et une planification soigneuse de l'ensemble.

A la base, l'ensemble est composé de trois éléments principaux: le temple du Cigognier, le théâtre qui lui fait face et une esplanade, espace apparemment vide, qui les sépare. Les deux monuments offrent des possibilités d'accès tant frontales que par l'arrière, qui desservent des parties différentes et bien délimitées de l'un et l'autre bâtiment. L'escalier traversant le mur de terrasse M7 devant la façade du théâtre, et les passages centraux ouverts dans le mur de scène, confirment l'existence d'une connexion rectiligne et axiale entre la cour du temple du Cigognier d'une part, et la scène, l'orchestra et la prohedria du théâtre de l'autre. Dans le secteur du Lavoëx, au sud-ouest du complexe, plusieurs édifices ont été construits au dernier tiers du $\mathrm{II}^{\mathrm{e}}$ siècle apr. J.-C., dont certains ont été manifestement incorporés à l'ensemble Cigognier/théâtre. Cet élargissement du complexe semble être accompagné d'une délimitation du secteur par rapport à un "extérieur» profane.

La contribution essaye d'approfondir quelques-uns de ces aspects, notamment les questions de l'organisation, des chemins de circulations, des axes de vue, etc. 
Voir notamment:

Matter, Georg, Das römische Theater von Avenches/Aventicum. Architektur, Baugeschichte, kulturhistorische Aspekte, Lausanne, Cahiers d'archéologie romande, 2009 (Cahiers d'archéologie romande 114, Aventicum XV).

Alain BADIÉ, Trois théâtres de Narbonnaise: Marseille, Toulouse et Orange

Les trois théâtres abordés permettent d'examiner trois situations méthodologiques très différentes.

Du théâtre de Toulouse aucun vestige n'est actuellement visible. C'est donc essentiellement à partir d'une documentation recueillie à la fin du $\mathrm{XIX}^{\mathrm{e}}$ siècle qu'il faut réfléchir.

Les vestiges du théâtre de Marseille sont quant à eux extrêmement lacunaires et les récentes fouilles de sauvetage menées par Ph. Mellinand (INRAP) n'ont révélé que quelques tranchées de récupération des murs de la cavea.

Le théâtre d'Orange est beaucoup mieux conservé que les deux précédents et particulièrement son bâtiment de scène qui s'élève toujours jusqu'à $36 \mathrm{~m}$ de hauteur. Malgré leurs différents états de conservation, ces trois exemples permettent de mieux comprendre l'intégration urbaine et l'architecture de ce type de monument.

\section{Voir notamment:}

Badié, Alain et Moretti, Jean-Charles, «Le théâtre de Marseille: un théâtre grec d'époque augustéenne", in D'Orient et d'Occident. Mélanges offerts à Pierre Aupert, dir. Alain Bouet, Bordeaux, Ausonius éditions, 2008, p. 245-256.

BADIÉ, Alain, «Le plan du théâtre antique de Toulouse: problèmes de restitution", in Tolosa. Nouvelles recherches sur Toulouse et son territoire dans l'Antiquité, dir. Jean-Marie Pailler, Rome, 2002, p. 233-241 (Collection de l'Ecole Française de Rome 281).

Badié, Alain, Moretti, Jean-Charles, Rosso, Emmanuelle, Tardy, Dominique, Le théatre antique d'Orange, Paris, Connaissance des Arts, H. S. 197, 2003. 